\title{
A clinical trial and molecular study of photoadaptation in vitiligo
}

\author{
C.L. Hexsel, ${ }^{\star}$ B.H. Mahmoud, ${ }^{\star}$ D. Mitchell, $\dagger$ J. Rivard, ${ }^{\star}$ M. Owen, ${ }^{\star}$ F.M. Strickland, ${ }^{\star} \ddagger$ H.W. Lim* \\ and I. Hamzavi* \\ *Multicultural Dermatology Center, Department of Dermatology, Henry Ford Hospital, Detroit, MI 48202, U.S.A. \\ $\dagger$ The University of Texas M.D. Anderson Cancer Center, Smithville, TX, U.S.A. \\ $\$$ Department of Internal Medicine - Rheumatology, University of Michigan, Ann Arbor, MI, U.S.A.
}

\section{Summary}

\section{Correspondence \\ Iltefat Hamzavi. \\ E-mail: ihamzav1@hfhs.org}

\section{Accepted for publication \\ 5 September 2008}

\section{Key words}

cyclobutane pyrimidine dimers, photoadaptation, phototherapy, ultraviolet $B$, vitiligo

\section{Conflicts of interest}

I.H. has received funding from Theralight for previous studies, but not for this particular study.

A brief communication report with results of the pilot phase of this study has been published: Rivard J, Hexsel C, Owen M et al. Photoadaptation of vitiliginous skin to targeted ultraviolet B phototherapy. Photodermatol Photoimmunol Photomed 2007; 23:258-60. Preliminary data from this study were presented as podium presentations at the 17th Annual Meeting of the Photomedicine Society, San Antonio, TX, held in January 2008.

ClinicalTrials.gov ID number: NCT00367224. DOI 10.1111/j.1365-2133.2008.08943.x
Background Photoadaptation to ultraviolet (UV) B phototherapy is due to both pigmentary and nonpigmentary influences.

Objectives To measure photoadaptation in vitiliginous skin and to compare it with normal pigmented skin.

Methods Seventeen patients with Fitzpatrick skin phototypes III-VI with vitiligo received six to nine UVB treatments, two to three times weekly. Minimal erythema dose (MED) testing was done at baseline and after all treatments; the percentage change in MED was analysed as a measure of photoadaptation. The percentage decrease in cyclobutane pyrimidine dimers (CPDs) over $24 \mathrm{~h}$ after a single exposure of 1 MED was analysed on vitiliginous and normal skin.

Results The mean \pm SD percentage change in MED from before to after treatments was: treated vitiliginous skin $28.5 \pm 39.9 \%(P=0.015)$, treated normal skin $35.9 \pm 49.9 \% \quad(\mathrm{P}=0.015)$, untreated vitiliginous skin $11.9 \pm 22.6 \% \quad(\mathrm{P}=$ $0 \cdot 070)$, untreated normal skin $25 \cdot 1 \pm 41 \cdot 3 \%(P=0.041)$. Of these patients, two-thirds had a positive percentage change in MED (photoadaptation). The mean amount of CPDs induced per megabase of DNA immediately after exposure was significantly higher in vitiliginous skin. The mean \pm SD percentage decrease in CPDs (rate of repair) in $24 \mathrm{~h}$ was $35.7 \pm 26 \cdot 8 \%$ in vitiliginous skin $(\mathrm{P}=0.027)$ and $46.2 \pm 19 \cdot 5 \%$ in normally pigmented skin $(\mathrm{P}=0.001)$; no difference was noted in the repair in vitiliginous skin compared with normal skin $(\mathrm{P}=0 \cdot 4)$

Conclusions Photoadaptation in vitiliginous and normal skin was observed in twothirds of patients. Vitiliginous skin had significantly more CPDs following UVB exposure; the rate of repair of UVB-induced DNA damage was equivalent to that in normal skin.
Vitiligo is characterized by destruction or inactivation of melanocytes, resulting in depigmented or hypopigmented macules and patches. ${ }^{1}$ A retrospective study showed that some patients with vitiligo treated with narrowband ultraviolet (UV) B radiation (NB-UVB) did not develop phototoxicity in their depigmented skin despite increasing doses of UV radiation. ${ }^{2}$ Thus, these patients underwent the phenomenon of photoadaptation or tolerance to NB-UVB radiation without repigmentation, a phenomenon frequently observed clinically during the course of phototherapy.

Photoadaptation was described by $\mathrm{Oh}$ et al. as follows: 'a number of changes occur that are adaptive, in the sense that they result in a diminished future response to equivalent doses of radiation'. ${ }^{3-5}$ Thus, subsequent equivalent UV radiation doses do not have the same effect on the skin as the first dose. This is the basis for the increasing UV radiation doses safely given to patients who undergo phototherapy.

Photoadaptation has been described in different Fitzpatrick skin phototypes and is thought to be due to both pigmentary and nonpigmentary influences. ${ }^{4,5}$ Studies have indicated that factors involved in photoadaptation include hyperkeratosis, acanthosis, melanogenesis, as well as an unknown factor yet to be identified which may be due to DNA repair or an immune-related process. ${ }^{5-10}$

Photoadaptation can be measured by several different methods, with different UV radiation sources and in different 
Fitzpatrick skin types. One of the methods is serial minimal erythema dose (MED) phototesting, as investigated by Gonzalez et al. ${ }^{11}$ in normal skin of a group of patients with psoriasis. The MED is defined as the minimum dose of UV radiation that causes visible erythema over the entire irradiated area after $24 \mathrm{~h}$. As a result, if over the course of phototherapy it takes a higher dose of UV radiation to cause erythema, then photoadaptation is considered to have taken place.

In phototherapy protocols, vitiliginous skin is always presumed to be equivalent to Fitzpatrick skin phototype I or II. On the other hand, there is no objective MED-based evidence to justify that conclusion. This is partly because of the lack of practical methods to measure MED within the depigmented skin, as the vitiliginous macules and patches frequently do not provide a large enough surface area to allow MED testing with whole-body phototherapy booths or solar simulators. In this study, using a light source that enables us to measure MED in a small body surface area, we measured both pigmentary and nonpigmentary photoadaptation in patients with vitiligo with the use of MED phototests during the course of UVB therapy.

Therefore, we designed a study with the purpose of measuring nonpigmentary photoadaptation to UVB phototherapy (vitiliginous skin) and to compare it with pigmentary photoadaptation (adjacent normally pigmented skin). In addition, we desired to investigate whether repair of UVB-induced DNA damage plays a role in photoadaptation by comparing the rate of repair of UVB-induced DNA damage between vitiliginous and normally pigmented skin.

\section{Materials and methods}

The study was reviewed and approved by the Investigational Review Board, Henry Ford Hospital (Detroit, MI, U.S.A.). Study procedures followed were in accordance with the ethical standards of the Institutional Review Board and with the Helsinki Declaration of 1975. Informed consent was obtained from all participants before the initiation of the study.

\section{Patients}

To be included in the study, patients had to be at least 18 years old and to have at least two symmetrical vitiliginous lesions on opposite sides of the body. Women who were lactating, pregnant or planning to become pregnant, and patients with serious systemic disease, immunosuppression and photosensitivity were excluded. Concomitant therapy for vitiligo during the study was prohibited, and patients were asked to discontinue psoralen + UVA and oral corticosteroid therapy for 4 weeks, and topical therapy and UVB phototherapy for 2 weeks, prior to the start of study treatments. The study was conducted throughout the calendar year.

\section{Photoadaptation factor}

Two bilateral symmetrical areas were selected, to avoid variation by body site, and were divided in four treatment sites: treated vitiliginous and normal skin on one side of the body, and untreated vitiliginous and normal skin on the contralateral symmetrical side.

To measure photoadaption, we performed MED phototests at baseline and after the completion of treatments in all four treatment sites: treated and untreated vitiliginous and normal skin.

To investigate the presence of photoadaptation, the following calculation was used to determine each patient's percentage change in MED from before to after treatments, or photoadaptation factor:

Photoadaptation factor $=\left[\left(\mathrm{MED}_{\text {final }}-\mathrm{MED}_{\text {initial }}\right) / \mathrm{MED}_{\text {initial }}\right] \times 100$.

Therefore, if after all treatments it took a higher dose of UVB to cause erythema, then photoadaptation had occurred.

\section{Phototherapy devices}

The first 10 patients were phototested and treated with targeted UVB phototherapy. As clinically NB-UVB is the first-line UV-based treatment for the management of vitiligo and is used more widely in clinical practice than targeted phototherapy, the subsequent seven patients were phototested and treated with NB-UVB.

\section{Targeted phototherapy}

The targeted UVB device used for phototesting and treatment of the first 10 patients is a mercury short-arc light source with a UVB handpiece with peaks at $302 \mathrm{~nm}$ and $312 \mathrm{~nm}$, with a weighted erythemal wavelength of $304 \mathrm{~nm}$ (Theralight, Carlsbad, CA, U.S.A.). ${ }^{12}$ The handpiece delivers UVB in a $1.9 \times 1.9 \mathrm{~cm}$ square area. The beam profile has a maximal variability of $3 \%$, corner to corner across the handpiece aperture (source: personal correspondence, Ray Hartman, Theralight). Although targeted, the spot size on the device was too large for phototesting of vitiliginous patches; thus, we placed oval slits in an opaque filter to decrease the spot size to approximately $1 \times 1.3 \mathrm{~cm}$ so that six spots would fit in the available phototested areas. For treatment purposes, the entire treated area was exposed to this radiation source.

\section{Narrowband ultraviolet B phototherapy}

The whole-body phototherapy unit used for the treatment of seven patients was the NB-UVB Ultralite 6809 Phototherapy unit (Lawrenceville, GA, U.S.A.), containing a bank of 48 fluorescent lamps (TL-100W/01; Philips, Eindhoven, Netherlands) with peak emission at 311-312 nm. As vitiliginous skin does not allow phototesting with a whole-body phototherapy unit due to the restricted surface area affected by vitiligo available, we used the hand-held NB-UVB Daavlin Dermapal unit (Bryan, OH, U.S.A.) comprising two fluorescent lamps (PL-S 9W/01/2P) with peak emission at 311-312 nm for MED phototesting of these patients with an appropriate template. 
The light output was measured using the Ultralite PH10AB integrating photometer.

Furthermore, in these seven patients the baseline MED was also determined using the Theralight targeted device; however, a second handpiece with a narrower spectrum, with reduced peak at $302 \mathrm{~nm}$ and a maximum peak at $312 \mathrm{~nm}$ with a weighted erythemal wavelength of $308 \mathrm{~nm}$, was used for this group of patients. This information was used for the study to determine the amount of cyclobutane pyrimidine dimers (CPDs) and the rate of repair of CPDs in patients treated with NB-UVB.

\section{Ultraviolet B phototherapy treatments}

In all patients, MED was determined on vitiliginous and normal skin sites, as described above. In the treated side of the symmetrical lesion, both vitiliginous and normal skin received six to nine treatments with UVB radiation with escalating doses two to three times weekly. All vitiliginous skin on the body was treated with NB-UVB, except for the 'untreated vitiliginous patch', which included a small area of surrounding normally pigmented skin. Starting doses were $70 \%$ of the MED, subsequently increased by $15 \%$ with each treatment. Treatment was withheld if severe erythema developed, decreased by $10 \%$ if moderate erythema occurred, left unchanged after mild symptomatic erythema and increased by $5 \%$ after mild asymptomatic erythema.

\section{Ultraviolet B-induced DNA damage}

After the last phototherapy treatment, a completely untreated area composed of vitiliginous and normal adjacent skin received a single treatment with targeted phototherapy: the dose used was the dose of the patient's vitiliginous skin baseline MED. Targeted UVB phototherapy was used in all patients and was selected for this determination due to its even beam profile, which allows for accuracy of the dose given. This treatment was followed by a total of four 4-mm punch biopsies: two biopsies immediately after the treatment (vitiliginous and normal skin), followed by two biopsies $24 \mathrm{~h}$ later (vitiliginous and normal skin). To ensure that no untreated gaps were biopsied and that the biopsy was performed on an evenly irradiated area, all targeted treated squares were marked with ink during treatment. These procedures were completed in the last nine patients. CPDs were measured both at baseline and at $24 \mathrm{~h}$ by radioimmunoassay (RIA). RIA is a very sensitive technique that determines the amount of CPDs induced per megabase of DNA (CPDs/mb). The change in UVBinduced DNA damage or CPDs in $24 \mathrm{~h}$ was assessed as a measure of rate of DNA repair.

\section{Radioimmunoassay}

For the RIA, 2 g of heat-denatured sample DNA was incubated with 5 pg of UV-irradiated poly $(\mathrm{dA})$; poly $(\mathrm{dT}$ ) (labelled to $>5 \times 10^{8}$ c.p.m. $\mu g^{-1}$ by nick translation with ${ }^{32} \mathrm{P}$-dTTP) in a total volume of $1 \mathrm{~mL}$ RIA buffer $\left(10 \mathrm{mmol} \mathrm{L}^{-1}\right.$ Tris, $\mathrm{pH} 7 \cdot 6$, $150 \mathrm{mmol} \mathrm{L}^{-1} \mathrm{NaCl}, 1 \mathrm{mmol} \mathrm{L}^{-1}$ ethylenediamine tetraacetic acid and $0 \cdot 15 \%$ gelatin; Sigma-Aldrich, St Louis, MO, U.S.A.). Antiserum was added at a dilution that yielded 30-60\% binding to ${ }^{32} \mathrm{P}$-labelled ligand, and after overnight incubation at $4{ }^{\circ} \mathrm{C}$, the immune complex was precipitated with goat antirabbit immunoglobulin (Calbiochem-Novabiochem, San Diego, CA, U.S.A.) and carrier serum from nonimmunized rabbits (UTMDACC, Science Park/Veterinary Division, Bastrop, TX, U.S.A.). After centrifugation, the pellet was dissolved in tissue solubilizer (NCS; Amersham Biosciences, Piscataway, NJ, U.S.A.), mixed with ScintiSafe (Fisher Scientific, Waltham, MA, U.S.A.) and the ${ }^{32} \mathrm{P}$ was quantified by liquid scintillation spectrometry. Under these conditions, antibody binding to an unlabelled competitor (the sample) inhibits antibody binding to the radiolabelled ligand. The details of the RIA and standards used for quantification of DNA damage have previously been described. ${ }^{13,14}$

\section{Data analysis}

Statistical inference and analysis consisted of different steps: first, determining the significance or P-values of the isolated percentage change in MED and CPDs in each of the four studied groups (treated and untreated vitiliginous and normal skin); second, analysing the significance of the comparison of the results between vitiliginous and normal skin.

The statistical significance of the percentage change in MED from before to after treatments, or photoadaptation factor, as well as the significance of the absolute and percentage change of $\mathrm{CPDs} / \mathrm{mb}$, was determined using one-sample t-tests. Onesample t-tests were also used for comparison of baseline (i.e. prior to any treatments) MEDs between skin selected to be treated and skin selected to be control or to be untreated, to determine how variable MEDs were on opposite sides of the body. Furthermore, one-sample t-tests were used to compare the percentage changes in MED and CPDs/mb between vitiliginous and normal skin. The distributional normality assumption of the t-test was adequately met for the data in question.

\section{Results}

Our sample consisted of 17 patients with Fitzpatrick skin phototypes III-VI: five (29\%) skin type III, three (18\%) skin type IV, five (29\%) skin type V and four (24\%) skin type VI; there were seven (41\%) men and $10(59 \%)$ women. The age at enrolment in the study ranged from 26 to 72 years (mean 48). Duration of disease at enrolment ranged from 6 to 42 years (mean 20). In two patients, a percentage change in MED was unable to be obtained. In one man with Fitzpatrick skin type VI, despite repeating phototestings on sequential days using multiple escalating doses, an MED was not able to be obtained. A woman with Fitzpatrick skin type V withdrew consent during the study. Of the 15 remaining patients, MED data from both treated and untreated skin was obtained from 14 patients. In one of the 15 patients, MED from vitiliginous skin in the control side was not completed because of 
insufficient skin area at the end to allow for the last MED testing to be completed. Thus, data from only the treated side in this patient were available for analysis.

There was no significant repigmentation of vitiliginous skin of the patients during the study, probably reflecting the small number of treatments delivered.

\section{Baseline minimal erythema doses}

Baseline MEDs were performed bilaterally, before any treatments were performed. We compared the mean percentage difference between the baseline MEDs between the site selected for treatment and the contralateral site selected for control (to be untreated), to determine how variable MEDs were on opposite sides of the body, and between vitiligininous and normal skin, in our sample, before any treatments. This comparison analysis used patients with data from all four settings $(\mathrm{n}=14)$.

The mean $\pm \mathrm{SD}$ percentage differences in baseline MEDs between site selected for treatment and the control site were as follow: $6 \cdot 3 \pm 24 \cdot 4 \%$ difference at baseline between vitiliginous site selected for treatment and the contralateral site selected for control (to be untreated) $(\mathrm{P}=0.3)$, and $2 \cdot 6 \pm$ $19 \%$ difference at baseline between normal skin site selected for treatment and the contralateral site selected for control (to be untreated) $(P=0 \cdot 6)$. These differences are not statistically significant.

As expected, at baseline, the MEDs on vitiliginous skin were significantly lower than MEDs on normal skin. At baseline, at site selected for treatment, the MEDs of vitiliginous skin were $22.9 \pm 23.5 \%$ (mean $\pm \mathrm{SD}$ ) lower than the MEDs on normal skin $(\mathrm{P}=0.003)$. In the control or to-be-untreated site, the MEDs of vitiliginous skin were $25 \cdot 1 \pm 24 \cdot 5 \%$ lower than the MEDs on normal skin ( $\mathrm{P}=0.002)$.

In patients who had MEDs and treatments with the targeted phototherapy handpiece with weighted erythemal wavelength at $304 \mathrm{~nm}$, the baseline MEDs in vitiliginous skin ranged from 120 to $570 \mathrm{~mJ} \mathrm{~cm}^{-2}$ and the baseline MEDs on normal skin ranged from 150 to $1320 \mathrm{~mJ} \mathrm{~cm}^{-2}$. In patients who had MEDs with the hand-held NB-UVB device, the baseline MEDs in vitiliginous skin ranged from 700 to $1400 \mathrm{~mJ} \mathrm{~cm}^{-2}$ and the baseline MEDs on normal skin ranged from 820 to $2200 \mathrm{~mJ} \mathrm{~cm}^{-2}$.

\section{Photoadaptation factor}

The percentage change in MED, from baseline to after treatments, of treated and untreated vitiliginous and normal skin, was calculated for each patient. As seen in Figure 1, with the exception of untreated vitiliginous skin, all mean percentage changes in MED are statistically significant and range from $25 \%$ to $35 \cdot 9 \%$.

Photoadaptation or percentage change in MED was not statistically different between vitiliginous and normal skin $(P=0 \cdot 5)$. However, normally pigmented skin had higher MEDs at baseline and thus, received higher initial doses of UV.

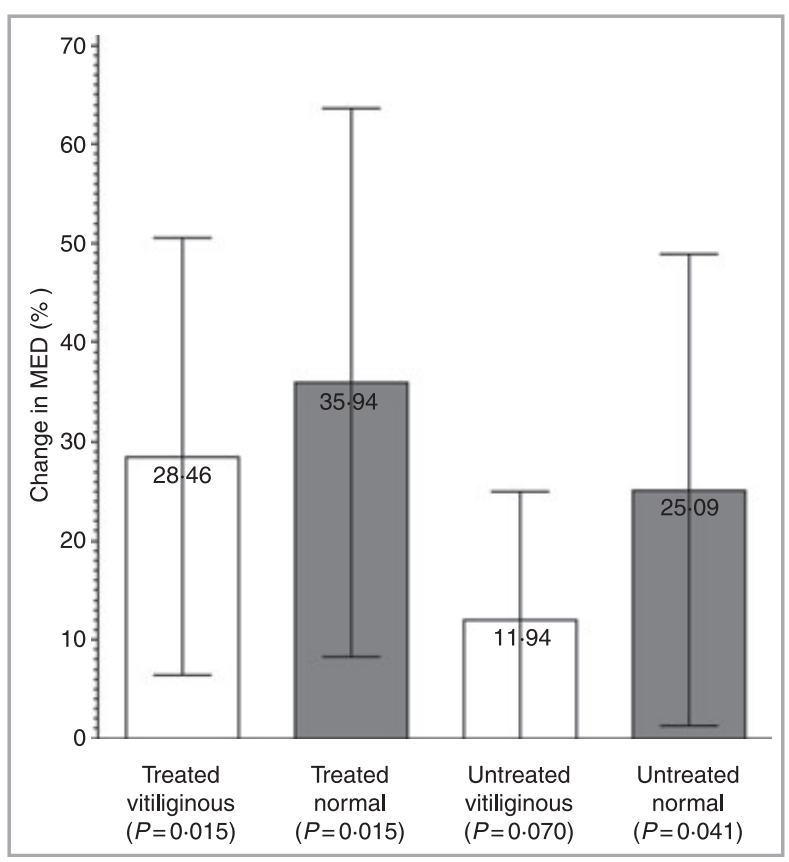

Fig 1. Photoadaptation factor [percentage change in minimal erythema dose (MED) from before to after treatments] in all patients. Results are shown as mean $\pm 95 \%$ confidence interval.

There was a substantial individual variation in the photoadaptive response. Interestingly, and of clinical and therapeutic relevance, we observed that one-third of the patients did not photoadapt. Figure 2a,b illustrates the patients who did and did not photoadapt, respectively. Figure 2a shows the photoadapters, or two-thirds of all the patients, who had a positive mean percentage change in MED from before to after treatments. Figure $2 \mathrm{~b}$ shows data from the nonphotoadapters, or one-third of the patients, in whom a negative mean percentage change in MED from before to after treatments was observed in treated skin.

\section{DNA damage and repair}

In order to understand photoadaptation at the molecular level, we measured the rate of repair or percentage reduction of UVB-induced DNA damage from baseline (immediately after one single treatment with $1 \mathrm{MED}$ ) to $24 \mathrm{~h}$, in both vitiliginous and normal skin in nine patients.

The rate of repair of UVB-induced DNA damage between vitiliginous and normal skin was calculated by the percentage of $\mathrm{CPDs} / \mathrm{mb}$ cleared within $24 \mathrm{~h}$. The rate of repair was $35.7 \%(\mathrm{P}=0.027)$ in vitiliginous skin (reduction from $52 \cdot 2$ to $32.7 \mathrm{CPDs} / \mathrm{mb})$ and $46 \cdot 2 \%(\mathrm{P}=0.001)$ in normally pigmented skin (reduction from $27 \cdot 1$ to $13 \cdot 4 \mathrm{CPDs} / \mathrm{mb}$ ).

Although the absolute numbers of CPDs/mb were significantly higher in vitiliginous skin, both at baseline $(52.2 \mathrm{com}-$ pared with $27 \cdot 1$ in normally pigmented skin, $P=0.008)$ and at $24 \mathrm{~h}(32 \cdot 7$ compared with 13.4 in normally pigmented skin, $\mathrm{P}=0 \cdot 004)$, the rate of repair of UVB-induced DNA damage/percentage clearance CPDs in $24 \mathrm{~h}$ was not significantly 


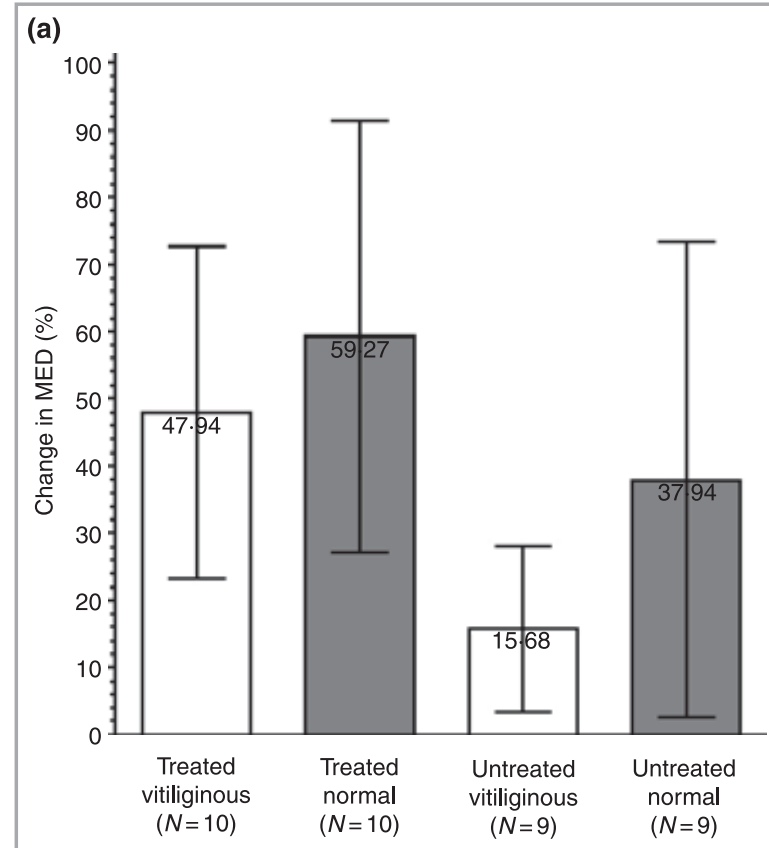

(b)

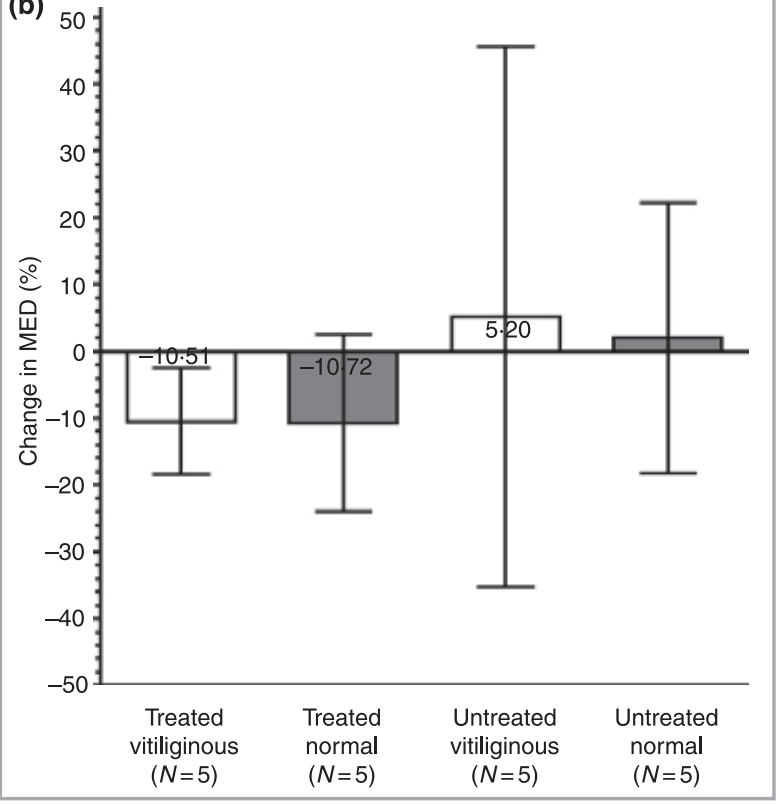

Fig 2. (a) Photoadaptation factor [percentage change in minimal erythema dose (MED) from before to after treatments] (a) in photoadapters and (b) in nonphotoadapters. Results are shown as mean $\pm 95 \%$ confidence interval.

different between vitiliginous and normal skin (mean \pm SD $35 \cdot 7 \pm 26 \cdot 8 \%$ in vitiliginous skin and $46 \cdot 2 \pm 19 \cdot 5 \%$ in normally pigmented skin, respectively, $\mathrm{P}=0 \cdot 4$ ); thus in absolute numbers, more CPDs/mb were repaired in vitiliginous skin.

\section{Discussion}

Our data provide objective evidence that photoadaptation occurs in depigmented vitiliginous skin, in the absence of significant repigmentation. We observed considerable individual variability in the ability of vitiliginous skin to photoadapt, as frequently observed in clinical practice. The degree of photoadaptation, reflected in percentage change in MED, of vitiliginous skin compared with normal pigmented skin was not statistically different, although normally pigmented skin had higher baseline MEDs and therefore was started on higher initial doses of UV. Nonphotoadapters did not adapt in either pigmented or depigmented skin, indicating that photoadaptive capacity may be genetically predetermined, and is only partially related to the intensity of skin pigment. Our results support the findings of Palmer et al. who measured photoadaptation in Fitzpatrick skin phototypes I-IV in 352 patients with psoriasis. Their study demonstrated that individuals with darker skin types photoadapted approximately equally per physical unit of UV radiation compared with individuals with lighter skin types, but those of darker skin types had greater photoadaptation in absolute terms because they were able to tolerate a higher initial dose of UV radiation. ${ }^{4}$

In our study, in addition to photoadaptation in both treated vitiliginous and normal skin, photoadaptation of untreated normally pigmented skin of patients with vitiligo was also observed, but not of untreated vitiliginous skin. We initially observed photoadaptation of untreated normally pigmented skin in the pilot phase of our study. ${ }^{5}$ Surprisingly, this observation was also made in this follow-up study using a larger number of patients, despite strict precautions taken afterwards to avoid phototesting of previously phototested areas by mapping each MED phototested area with transparencies. A report of an increase in the number of melanocytes in both UVB irradiated and nonirradiated control areas, when compared with baseline levels, following UVB radiation, was previously described. ${ }^{15}$ Whether this postulated systemic effect of pigmentation accounts for the observed photoadaptation of untreated normally pigmented skin remains unclear.

DNA repair has been hypothesized to play a role in nonpigmentary photoadaptation..$^{5-10}$ Previous work from Yarosh suggested that there is a direct relationship between CPDs and erythema. ${ }^{16}$ Moreover, Bataille et al. previously studied the formation of CPDs throughout the course of 18 phototherapy treatments in patients with psoriasis; they observed that CPD formation reached a plateau after three UVB treatments, and decreased following subsequent exposures despite exposure to increasing doses of UVB. The authors concluded that the decrease in CPDs may be due to the phenomenon of photoadaptation. ${ }^{7}$

We aimed to investigate whether repair of UVB-induced DNA damage plays a role in photoadaptation. We compared CPD formation and repair between vitiliginous and normal skin after a single exposure of 1 MED. CPD formation was statistically higher in vitiliginous skin. On the other hand, the rate of repair of CPDs was not statistically different between vitiliginous and normal skin. As vitiliginous skin had higher baseline UVB-induced DNA damage or CPDs/mb, a larger absolute number of CPDs was repaired in vitiliginous skin compared with normally pigmented skin. This retained DNA repair capacity, in conjunction with retained photoadaptive 
capacity, suggests that vitiliginous skin retains some photobiological properties of unaffected skin despite the loss of pigment. This may be of therapeutic interest as vitiliginous skin is currently widely treated by phototherapy as Fitzpatrick skin type I, irrespective of the patient's Fitzpatrick skin type of the unaffected skin. The clinical relevance of our findings is that it is conceivable that one can design a more aggressive phototherapy treatment protocol for the treatment of vitiligo. Further research is needed to determine if this would be efficacious and beneficial for patients with different photoadaptive capacities.

To the best of our knowledge, this is the first time that photoadaptation of vitiliginous skin has been demonstrated. There are limitations to our analysis. We did not have any patients with vitiligo whose constitutive skin types are of Fitzpatrick type I and II. While we performed MEDs on vitiliginous skin in this proof-of-principle study, we recognize that in practice, it is not practical to do so because of the small area of vitiliginous patches that many patients present with. Lastly, we used both targeted phototherapy and NB-UVB; because of the small number of patients, our study did not have the power, based on the minimal effect size of interest determined using a two-sided alpha level of $0 \cdot 05$, to draw separate conclusions on NB-UVB and targeted phototherapy.

In conclusion, despite lack of significant repigmentation, two-thirds of the patients with vitiligo in our study developed photoadaptation. The photoadaptive capacity and the rate of repair of UVB-induced DNA damage were equivalent between vitiliginous and normal skin. Therefore, vitiliginous skin appears to retain some of the important baseline photobiological properties of normally pigmented skin. Further research is needed to determine if patients with different photoadaptive capacities would benefit from more aggressive UVB treatment protocols.

\section{Acknowledgments}

The work on this project was supported by the C.S. Livingood Fund from the Department of Dermatology of the Henry Ford Hospital, and resources from the University of Texas M.D. Anderson Cancer Center.

\section{References}

1 Kovacs SO. Vitiligo. J Am Acad Dermatol 1998; 38:647-66.

2 Hamzavi I, Deleon S, Yue K, Murakawa G. Repigmentation does not affect tolerance to NB-UVB light in patients with vitiligo. Photodermatol Photoimmunol Photomed 2004; 20:117 (Abstract).

3 Oh C, Hennessy A, Ha T et al. The time course of photoadaptation and pigmentation studied using a novel method to distinguish pigmentation from erythema. J Invest Dermatol 2004; 123:965-72.

4 Palmer RA, Aquilina S, Milligan PJ et al. Photoadaptation during narrowband ultraviolet-B therapy is independent of skin type: a study of 352 patients. J Invest Dermatol 2006; 126:1256-63.

5 Rivard J, Hexsel C, Owen M et al. Photoadaptation of vitiliginous skin to targeted ultraviolet B phototherapy. Photodermatol Photoimmunol Photomed 2007; 23:258-60.

6 Lock-Andersen J, Therkildsen P, de Fine Olivarius F et al. Epidermal thickness, skin pigmentation and constitutive photosensitivity. Photodermatol Photoimmunol Photomed 1997; 13:153-8.

7 Bataille V, Bykov VJ, Sasieni P et al. Photoadaptation to ultraviolet (UV) radiation in vivo: photoproducts in epidermal cells following UVB therapy for psoriasis. Br J Dermatol 2000; 143:477-83.

8 Hamzavi I. Photoadaptation: a path toward rational phototherapy protocols. J Invest Dermatol 2006; 126:2156-8.

9 Hennessy A, Oh C, Rees J, Diffey B. The photoadaptive response to ultraviolet exposure in human skin using ultraviolet spectrophotometry. Photodermatol Photoimmunol Photomed 2005; 21:229-33.

10 Mitchell DL, Greinert R, de Gruijl FR et al. Effects of chronic lowdose ultraviolet $\mathrm{B}$ radiation on DNA damage and repair in mouse skin. Cancer Res 1999; 59:2875-84.

11 Gonzalez S, Hegyi V, Baqer A et al. Development of cutaneous tolerance to ultraviolet B during ultraviolet B phototherapy for psoriasis. Photodermatol Photoimmunol Photomed 1996; 12:73-8.

12 Asawanonda P, Chingchai A, Torranin P. Targeted UV-B phototherapy for plaque-type psoriasis. Arch Dermatol 2005; 141:1542-6.

13 Mitchell DL. Radioimmunoassay of DNA damaged by ultraviolet light. In: Technologies for Detection of DNA Damage and Mutations (Pfeifer G, ed.). New York: Plenum Publishing Corp, 1996; 73-85.

14 Mitchell DL. Quantification of DNA photoproducts in mammalian cell DNA using radioimmunoassay. In: Methods in Molecular Biology: DNA Repair Protocols (Henderson DS, ed.). Totowa, NJ: The Humana Press Inc, 2006; 239-49.

15 Stierner U, Rosdahl I, Augustsson A, Kagedal B. UVB irradiation induces melanocyte increase in both exposed and shielded human skin. J Invest Dermatol 1989; 92:561-4.

16 Yarosh DB. DNA repair, immunosuppression, and skin cancer. Cutis 2004; 74:10-13. 